\title{
Green Driving Optimization of a Series Hybrid Electric Vehicle
}

\author{
Roberto Lot and Simos A. Evangelou
}

\begin{abstract}
This paper develops an indirect optimal control methodology to achieve green driving optimisation for series hybrid electric vehicles. Starting from a given vehicle mission, specified in terms of a road journey that has to be completed in a given amount of time, the power sharing among the powertrain sources and the vehicle speed profile along the journey are optimised and found. The scheme combines parametric modelling of the vehicle and powertrain together with computationally efficient optimal control software to provide an optimization strategy that works in real-time. Simulation results that demonstrate the success of the method and provide further insight into efficient driving, are presented.
\end{abstract}

\section{INTRODUCTION}

Hybrid Electric Vehicle (HEV) powertrains are more complex than their counterparts in conventional vehicles. The additional powertrain 'degrees of freedom' allow optimisation to take place at two different levels: firstly, at architecture level, and secondly, at control level. The main relevant control objective is to provide energy management to reduce fuel consumption and exhaust emissions, while maintaining or even increasing vehicle performance. For this purpose, Supervisory Control Systems (SCSs) are used to decide intelligently on how to provide energy to satisfy the vehicle load from the multiple energy sources existing in HEV powertrains. A wide range of SCSs, from rule-based to optimisation-based, have been proposed in the literature [1]-[6]. Many global optimisation-based approaches involve significant amount of computation and are therefore not implementable in real-time, while instantaneous optimisationbased techniques such as the Equivalent Consumption Minimization Strategy and its variants have a reduced computational burden but are not as accurate [7]-[9].

This paper presents a global optimisation-based SCS that utilises indirect optimal control techniques on the powertrain energy flow to achieve fuel consumption minimisation for a given vehicle mission. In standardised tests such missions are defined in terms of a given speed profile, however in the present work they are specified in terms of a route which has to be completed in a predetermined amount of time. Therefore, not only does the method optimize the powertrain energy flow, but also it computes the best speed profile along the route, complying with driving safety and comfort requirements.

R. Lot is with the Department of Industrial Engineering, University of Padova, 35100 Padova, Italy; roberto . lot @unipd. it

S. A. Evangelou is with the Departments of Electrical and Electronic, and Mechanical Engineering, Imperial College London, UK; s.evangeloulimperial.ac.uk
Similar type of optimisation problems have been studied extensively in the literature in the context of finding road vehicle speed trajectories for minimum fuel consumption, with some of the early work described in [10], [11]. There are also numerous studies on the subject of train operation where optimal speed reference trajectories are calculated and planned to fulfil a number of objectives, such as minimising energy consumption, enabling punctuality, achieving safe and reliable operation, and providing comfortable driving; see the survey in [12]. Various methods have been employed to solve these optimisations, including nonlinear programming and dynamic programming [13], while problems involving trains with regenerative capability, with simultaneous optimisation of the State of Charge of the energy storage devices, have also been considered [14]. Additionally, velocity trajectory optimisation has been investigated in the context of conventional trucks [15] (weighted journey time and fuel consumption criterion) and hybrid trucks [16]. The starting point in [16] is the corresponding optimisation of a conventional vehicle under constant load conditions and fixed journey time, for which the optimal speed profile comprises maximum acceleration, followed by a constant speed segment, then coasting and finally maximum deceleration [17] - similar to the pulse-and-gliding manoeuvre identified in [18] in the context of fuel-optimal driving of passenger cars in car-following scenarios. Approximately, this speed profile shape is assumed to be the optimal solution in the case of hybrid trucks also, although this is not necessarily true since hybrid vehicles can recover kinetic energy while braking or descending an incline. Algebraic expressions for the equivalent fuel consumption and travelling time for each speed profile segment are found and optimised together subject to the given constraints, using nonlinear programming, to find the length of each segment. Apart from the speed trajectory the optimisation calculates the power trajectory, which can subsequently be used to optimise the power split between primary and secondary energy sources in the powertrain.

The contribution of the SCS developed in this paper results from its main features: it does not presuppose any specific shape for the optimal solution, instead it simultaneously optimises fuel consumption, power split and speed profile; it is developed and tested on a symbolic dynamic vehicle model that maintains an appropriate balance between complexity and accuracy of transient behaviour; and, it utilises computationally efficient optimal control software [19] with excellent convergence speed appropriate for real-time application.

In the next section the model of the vehicle and its powertrain is introduced. Section III establishes the green 


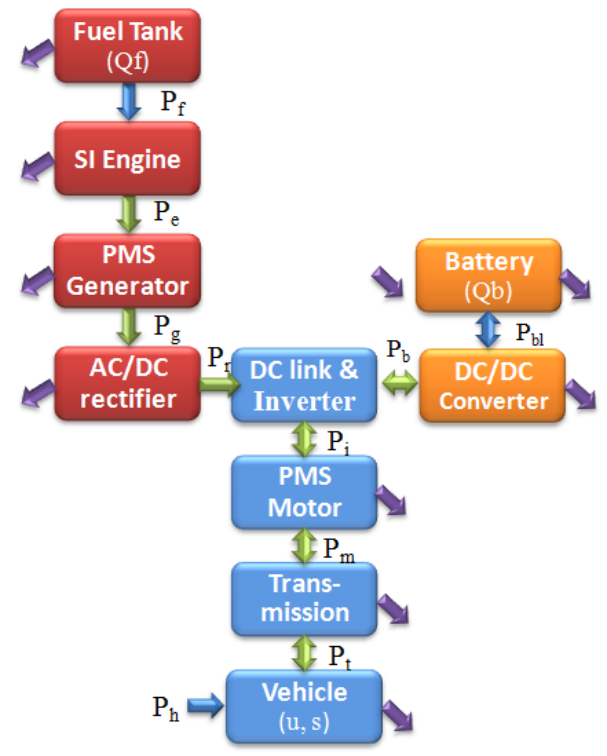

Fig. 1. Powertrain architecture of a Series Hybrid Electric Vehicle (purple arrows indicate power losses).

driving optimisation problem. Section IV presents results where the optimal speed profile and power flow are discussed. Conclusions are given in Section V.

\section{Series Hybrid Electric Vehicle Model}

This work focuses on the energy management optimization of a Series Hybrid Electric Vehicle (S-HEV). The S-HEV powertrain architecture is depicted in Figure 1 and consists of three branches: the spark ignition (SI) engine, the battery and the permanent magnet synchronous (PMS) motor branch.

In thrusting operating conditions, i.e. at constant speed or while accelerating, power is request to drive the vehicle. The SI engine is fed by the fuel tank and transforms fuel chemical power $P_{f}$ into mechanical power $P_{e}$. The SI engine is directly connected to the PMS generator, which converts $P_{e}$ into the electric AC power $P_{g}$ to supply the rectifier. The rectifier converts this to DC electric power $P_{r}$ at constant voltage $v_{d c}$ and provides it to the DC link. In the other branch, the battery (possibly) provides some additional power $P_{b l}$ to the DC/DC converter, which steps up the battery voltage to $v_{d c}$ and provides power $P_{b}$ to the DC link. The overall DC link power $P_{r}+P_{b}$ is converted from DC into AC by the inverter, which provides the electric power $P_{i}$ to the PMS motor. This electric power is then converted into mechanical power $P_{m}$, which is given to the transmission that conveys the power to the wheels, and the vehicle is finally driven with a power $P_{t}$. In any of the power conversion processes, there is some power loss which is represented in the figure by purple arrows.

To decelerate the vehicle, mechanical brakes are actuated and the corresponding power $P_{h}$ extracted is converted into heat and dissipated. At the same time, it is also possible (and convenient) to recovering some energy, by conveying braking power through the transmission up to the battery.

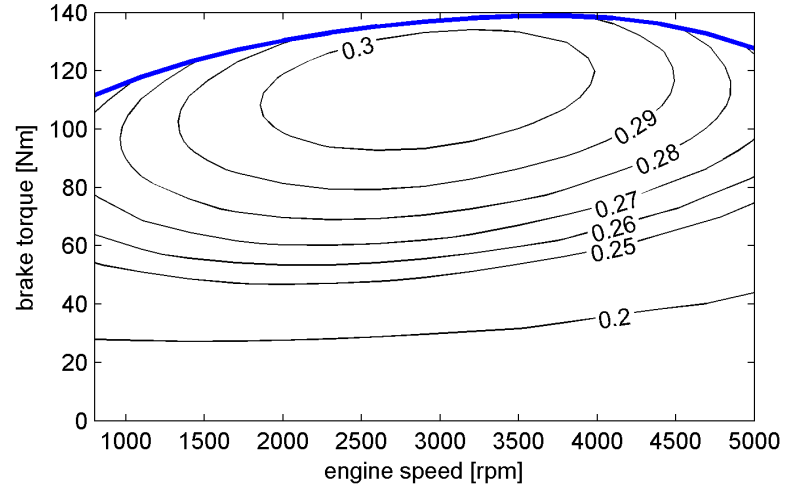

Fig. 2. Efficiency of the spark ignition engine.

Additionally, the battery may be recharged by using a fraction of the generator power.

In summary, the S-HEV vehicle has three independent sources of power $P_{b}, P_{g}$, and $P_{h}$ (corresponding respectively to the battery, generator, and brakes), that may be variously combined to obtain the desired values of vehicle speed and acceleration. This redundancy may be effectively exploited to reduce the fuel consumption by means of a proper power management strategy.

\section{A. Combustion engine branch}

1) Spark Ignition Engine: the operation of the SI engine is governed by complex physical processes which are difficult to model. However, these processes are fast as compared to the events of interest in the present work, therefore average modelling of this component is adopted. Thus the engine efficiency is given as a function of the rotational speed $\omega_{e}$ and brake torque $T_{b}$ :

$$
\eta_{e}=\eta_{e}\left(\omega_{e}, T_{b}\right)
$$

Figure 2 shows the steady state efficiency map of a hypothetical, 2L engine [20], [21], which has a maximum efficiency $\eta_{e, \max }=0.308$ for an brake torque of $115 \mathrm{Nm}$ at $2860 \mathrm{rpm}$. Starting from this operating point, the efficiency decreases as the brake torques increases mainly because the fuel/air ratio increases over its stoichiometric value, while the efficiency also decreases for lower brake torques because the engine is throttled. From the optimal operating point, the efficiency also decreases when the engine speed increases mainly because of the increment of pumping losses, while the efficiency also decreases when the engine speed decreases mainly because of the reduction of thermodynamic efficiency with speed.

2) Permanent Magnet Synchronous Generator: PMS machines combine a number of attractive features when used in hybrid vehicle applications, such as higher torque-to-inertia ratio and power density than those of induction or woundrotor synchronous machines. For these reasons a 3-phase star-connected PMS generator has been adopted. The dynamic electromagnetic behaviour of the PMS generator may be effectively described in the rotor $d-q$ reference frame 
TABLE I

Permanent Magnet Synchronous Machines Parameters

\begin{tabular}{lccc}
\hline Parameter & Symbol & Motor & Generator \\
\hline max torque & $T_{l}$ & $160 \mathrm{Nm}$ & $400 \mathrm{Nm}$ \\
max speed & $\omega_{\max }$ & $5000 \mathrm{rpm}$ & $5000 \mathrm{rpm}$ \\
max current & $I_{\max }$ & $150 \mathrm{~A}$ & $250 \mathrm{~A}$ \\
stator resistance & $R$ & $31 \mathrm{~m} \Omega$ & $60 \mathrm{~m} \Omega$ \\
stator inductances & $L_{d}, L_{q}$ & $0.17 \mathrm{mH}$ & $0.045 \mathrm{mH}$ \\
rotor magnetic flux & $\lambda$ & $0.13 \mathrm{~Wb}$ & $0.20 \mathrm{~Wb}$ \\
number of pole pairs & $p$ & 6 & 6 \\
\hline
\end{tabular}

[22] (where direct components $d$ are aligned with the magnet flux, while $q$ quadrature components are orthogonal to this flux) by the following non-linear differential equations:

$$
\begin{aligned}
L_{d g} \frac{d}{d t} i_{d g} & =v_{d g}-R_{g} i_{d g}+p_{g} \omega_{g} L_{q g} i_{q g} \\
L_{q g} \frac{d}{d t} i_{q g} & =v_{q g}-R_{g} i_{q g}-p_{g} \omega_{g}\left(L_{d g} i_{d g}+\lambda_{g}\right)
\end{aligned}
$$

where $i_{d g}, v_{d g}$ and $i_{q g}, v_{q g}$ are the direct and quadrature components of stator currents and voltages respectively, $\omega_{g}$ is the rotor angular speed, while the other parameters are described in Table I (for the generator append subscript $g$ to the symbols in the table). Equations (2) model only the power losses due the resistance $R_{g}$ of stator copper windings, while actually there are other electromagnetic dissipation sources [23] such as Eddy current losses $\left(\propto \omega_{g}^{2}\right)$ and hysteresis losses $\left(\propto \omega_{g}^{2}\right)$, as well as mechanical losses [23] such as bearing losses $\left(\propto \omega_{g}\right)$ and windage losses $\left(\propto \omega_{g}^{5}\right)$. These additional losses are modelled via a dissipation torque in the dynamic equation of the rotor as follows:

$$
J_{g} \frac{d}{d t} \omega_{g}=\frac{3}{2} p_{g} \lambda_{g} i_{q g}+T_{l g}+T_{d g}\left(\omega_{g}\right)
$$

where $J_{g}$ is the rotor inertia, $T_{l g}$ is the load torque, i.e. the SI engine torque, $T_{d g}\left(\omega_{g}\right)$ is the dissipation torque and $\frac{3}{2} p_{g} \lambda_{g} i_{q g}$ corresponds to the electromagnetic torque. The vector control strategy adopted for the generator uses a null direct current $i_{d g}=0$. To further simplify the model, it may be observed that the dynamics of electromagnetic phenomena are much faster than mechanical ones, hence transient currents may be neglected. The inertia torque $J_{g} \frac{d}{d t} \omega_{g}$ is neglected also, as in normal operating conditions this torque is reasonably smaller than the load torque. These assumptions lead to the simplification of differential equations (2), (3) into a set of steady-state algebraic equations. These equations may be easily solved in terms of currents and voltages, leading to the following expressions for input and output power:

$$
\begin{aligned}
& P_{e}=\omega_{g} T_{l g} \\
& P_{g}=\omega_{g}\left(T_{l g}+T_{d g}\right)-\frac{2}{3} R_{g} \frac{\left(T_{l g}+T_{d g}\right)^{2}}{\left(p_{g} \lambda_{g}\right)^{2}}
\end{aligned}
$$

Therefore the generator efficiency:

$$
\eta_{g}=\frac{P_{g}}{P_{e}}
$$

may be explicitly evaluated as a function of the load torque $T_{l g}$ and velocity $\omega_{g}$, as shown in Figure 3 . The figure also

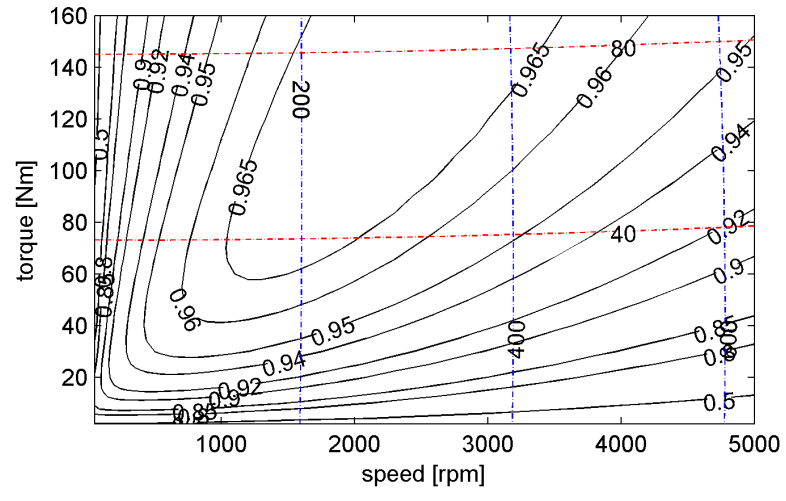

Fig. 3. Efficiency (solid lines), current (horizontal dashed lines) and voltage (vertical dashed lines) of the PMS Generator.

shows the current $i_{q g}$, which is roughly proportional to the torque, and the overall voltage $\sqrt{v_{d g}^{2}+v_{q g}^{2}}$, which is roughly proportional to the speed. The PMS generator efficiency is very high in a wide range of operating conditions, even if it is very poor at low speeds, where the resistance losses $R_{g} i_{q g}^{2}$ are predominant, and at low torques, where the mechanical losses $\omega_{g} T_{d g}\left(\omega_{g}\right)$ are predominant.

3) Rectifier: The rectifier converts the generator $\mathrm{AC}$ into DC at constant voltage $v_{d c}=700 \mathrm{~V}$. A pulse width modulated (PWM) rectifier is used [24] in which the high frequency switching dynamics can be neglected by averaging them out [25]. We are essentially interested in the efficiency of the energy transformation, which is simply modelled by means of a constant efficiency factor $\eta_{r}=0.96$. .

\section{B. Battery branch}

1) Battery: The battery dynamics is simply described by the following differential equation:

$$
\frac{d}{d t} Q_{b}=-i_{b}
$$

where $Q_{b}$ is the actual battery charge and $i_{b}$ is the battery current, assumed positive during the discharge phase. Moreover, the battery power is:

$$
P_{b l}=i_{b} v_{b}
$$

where $v_{b}$ is the closed circuit voltage of the battery, which depends both on the battery charge $Q_{b}$ and current $i_{b}$. A Li-ion battery model based on the work presented in [26], [27] is used. This model expresses the electrochemical parameters of the battery directly in terms of parameters of an equivalent electrical circuit, demonstrating that the Liion battery voltage may be approximated with the following expression:

$$
v_{b}=E_{b}-R_{b} i_{b}=E_{0}+\left(1-\frac{Q_{\max }}{Q}\right)+A e^{B\left(Q-Q_{\max }\right)}-R_{b} i_{b}
$$

where $E_{b}$ is the open circuit voltage, $R_{b}$ is the internal resistance, $E_{0}$ is the nominal voltage, $Q_{\max }$ is the capacity, and $A, B$ are two additional constants. 


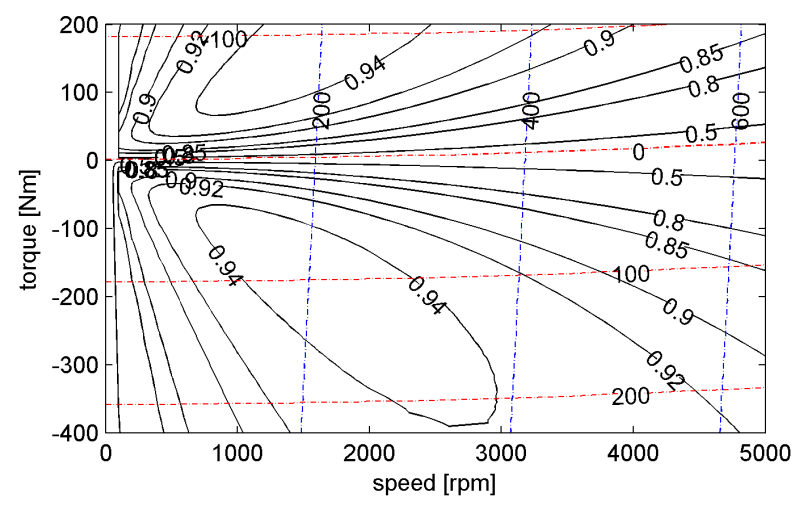

Fig. 4. Efficiency of the reversible PMS machine (generator $=$ positive torque, motor $=$ negative torque).

2) DC/DC converter: It increases the battery voltage to $v_{d c}$. The DC/DC converter, similarly to the rectifier, is simply modelled as a static element having constant efficiency $\eta_{d c}=0.96$. Since the converter is bi-directional, the power conversion may be described by means of the following equation:

$$
P_{b}=\eta_{d c}^{\operatorname{sign}\left(P_{b}\right)} P_{b l}
$$

where $P_{b l}$ is the battery power on the low voltage side, while $P_{b}$ is the battery power on the DC link side. The efficiency is adjusted according to the direction of the power flow, i.e. when positive power flows from the battery to the DC link $P_{b}=\eta_{d c} P_{b l}$, on the contrary when negative power flows from the DC link to charge the battery $P_{b l}=\eta_{d c} P_{b}$.

\section{Transmission branch}

1) Inverter: a bidirectional pulse width modulated (PWM) inverter is adopted. Similarly to the rectifier, it is simply modelled by means of a constant efficiency factor $\eta_{i}=0.96$. The power balance of the DC link and inverter is described by the following equation:

$$
P_{i}=\eta_{i}^{\operatorname{sign}\left(P_{r}+P_{b l}\right)}\left(P_{r}+P_{b l}\right)
$$

where once again the efficiency is adjusted according to the direction of the power flow.

2) Permanent Magnet Synchronous Motor: Equations (2)(4) that describe the PMS generator may be adapted to describe also the reversible PMS motor/generator. For the latter machine, it is convenient to adopt the convention that when it works as a generator, power (as well as the load torque and quadrature current) are assumed positive, while when it works as a motor powers are assumed negative. Therefore the PMS motor/generator equations may be derived from (4) simply by replacing suffix $g$ with $m$, as follows:

$$
\begin{aligned}
P_{m} & =\omega_{m} T_{l m} \\
P_{i} & =\omega_{g}\left(T_{l m}+T_{d m}\right)-\frac{2}{3} R_{m} \frac{\left(T_{l m}+T_{d m}\right)^{2}}{\left(p_{m} \lambda_{m}\right)^{2}}
\end{aligned}
$$

where $P_{m}$ is the motor mechanical power, while $P_{i}$ is the inverter electric power. The motor efficiency:

$$
\eta_{m}=\left(\frac{P_{m}}{P_{i}}\right)^{\operatorname{sign}\left(P_{i}\right)}
$$

is shown in Figure 4 both for the motor (negative torque) and generator (positive torque) operating conditions.

3) Transmission: A transmission with constant ratio $\tau=$ 10 is used and hence the relation between the motor angular speed $\omega_{m}$ and the vehicle forward speed $u$ is simply:

$$
\omega_{m}=\tau u
$$

It is assumed that the transmission has a constant efficiency $\eta_{t}=0.96$. The bi-directional power flow is hence modelled with the following equation:

$$
P_{t}=\eta_{t}^{\operatorname{sign}\left(P_{m}\right)} P_{m}
$$

i.e. when positive power flows from the PMS motor to the vehicle $P_{t}=\eta_{t} P_{m}$, on the contrary when negative power flows from the vehicle to the battery $P_{m}=\eta_{t} P_{t}$.

4) Brakes: Mechanical brakes are simply modelled as power withdrawal, i.e a source of negative power $P_{h}$. This power is converted into heat and dissipated.

5) Vehicle: The gross motion of the vehicle is described in terms of longitudinal speed $u$ and yaw rate $\Omega$, by using the single-track, non-holonomic vehicle model depicted in Figure 5. The longitudinal dynamics is described by the following differential equation:

$$
m \frac{d}{d t} u=F_{u}-F_{t}-F_{D}
$$

where $m$ is the overall mass, $F_{t}$ the resistance force due to tires, $F_{D}=\frac{1}{2} \rho C_{D} A u^{2}$ the aerodynamics drag resistance and $F_{u}$ is the longitudinal driving force. The latter force is proportional to the transmission and brakes power as follows:

$$
F_{u}=\frac{P_{t}+P_{h}}{u}
$$

The travelled distance $s$ is estimated by integrating the longitudinal speed:

$$
\frac{d}{d t} s=u
$$

The non-sliding assumption for the front wheel leads to the following algebraic equation:

$$
w \Omega=u \tan \delta
$$

where $\delta$ is the steering angle and $w$ the wheelbase. The road is assumed to be flat, the curvature $\Theta$ of the road center line may be calculated from its cartesian coordinates $(x, y)$ as a function of the travelled distance $s$, according to the following expression:

$$
\Theta(s)=\sqrt{\left(\frac{d^{2} x}{d s^{2}}\right)^{2}+\left(\frac{d^{2} y}{d s^{2}}\right)^{2}}
$$

It is reasonable to assume that when driving on a single lane rural road, the rider remains approximately in the middle 


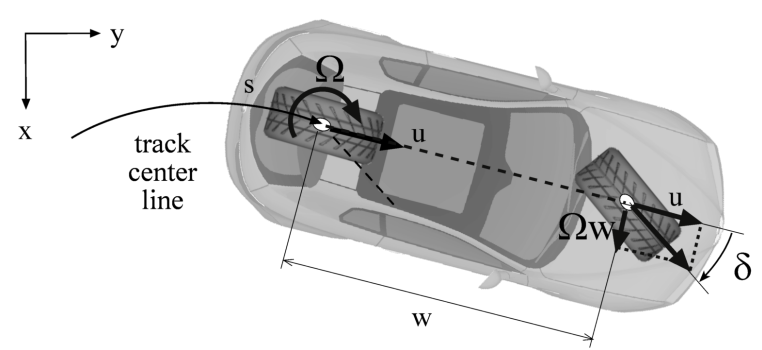

Fig. 5. Single-track, non holonomic, vehicle model.

of its lane. Therefore, the vehicle yaw rate $\Omega$ is simply proportional to the vehicle speed and road curvature:

$$
\Omega=u \Theta(s)
$$

This assumption is not representative of drivers' behavior at intersections, therefore sharp corners must be converted into smoother profiles by properly filtering the curvature $\Theta(s)$.

\section{Integration of model components}

Each element of the hybrid transmission has been modeled in a quite simple manner, however the interconnection of these elements leads to a S-HEV model which expresses a complex behavior, with a level of detail adequate to capture the system sensitivity to different power management strategies.

On the engine power branch, the efficiency $\eta_{f}$ of the transformation of fuel chemical power into electric power is simply the product of the engine and generator efficiencies:

$$
\eta_{f}=\eta_{e} \eta_{g}
$$

Moreover, the SI engine and the PMS generator are mechanically connected:

$$
\begin{aligned}
\omega_{e} & =\omega_{g} \\
T_{b} & =T_{l g}
\end{aligned}
$$

and hence efficiency $\eta_{f}$ is represented in Figure 6 as a function of the common speed and torque. It is important to remember that the SI engine is not mechanically connected to the vehicle wheels, hence a requested engine power may be supplied by freely choosing among different combinations of torque and speed. Among them it is obviously convenient to select the combination having greatest efficiency, highlighted in the figure by means of a dashed line. Once a control strategy that selects the most efficient operating points has been implemented, efficiency and output power become a function of the input power only, i.e a function of the fuel mass rate.In this case, it has been found that the relation between fuel mass rate $q_{f}$ and generator output power $P_{g}$ is approximately linear [16]:

$$
P_{g} \simeq \alpha_{f} Q_{H V}\left(q_{f}-q_{f 0}\right)
$$

where $Q_{H V}=44 \mathrm{MJ} / \mathrm{kg}$ is the gasoline heating value, $q_{f 0}=$ $9.1 \mathrm{mg} / \mathrm{s}$ is the fuel mass rate to keep the engine idle and the coefficient $\alpha_{f} \simeq 0.266$ it the marginal efficiency of the power

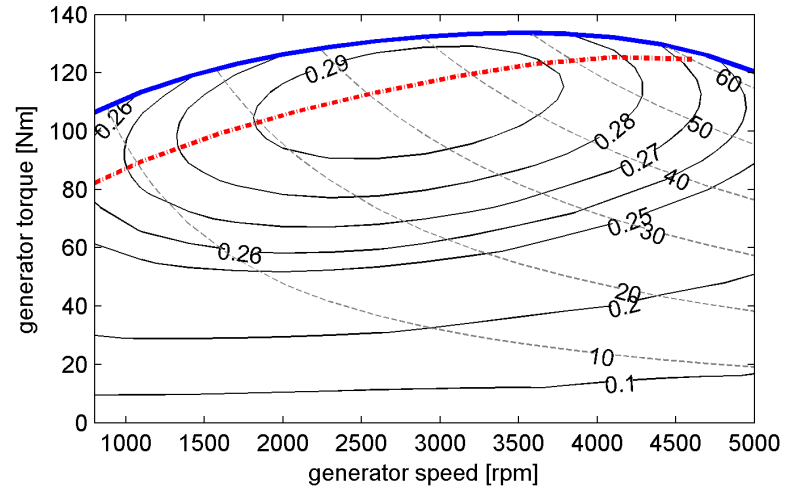

Fig. 6. Engine and generator joint operating characteristics: specific fuel consumption (solid black lines), output power (dotted gray lines) and most efficient operating points (thick dotted/dashed line).

transformation. The overall fuel transformation efficiency it finally:

$$
\eta_{f}=\frac{P_{g}}{Q_{H V} q_{f}} \simeq \frac{\alpha_{f} P_{g}}{P_{g}+\alpha_{f} Q_{H V} q_{f 0}}
$$

while the fuel mass rate may be rewritten as a function of the generator output power as follows:

$$
\frac{d}{d t} Q_{f}=q_{f} \simeq q_{f 0}+\frac{P_{g}}{Q_{H V} \alpha_{f}}
$$

By coupling and manipulating equations (9), (10), (12), (14), and (24), the vehicle power flow may be completely described as a function of three independent power sources $\mathbf{u}=\left\{P_{g}, P_{b}, P_{h}\right\}$, respectively the generator, battery, and brakes, as follows:

$$
\begin{aligned}
& P_{f}=\eta_{f}^{-1} P_{g} \\
& P_{r}=\eta_{r} P_{g} \\
& P_{b l}=\eta_{d c}^{-\operatorname{sign} P_{b}} P_{b} \\
& P_{i}=\eta_{i}^{\operatorname{sign}\left(\eta_{r} P_{g}+P_{b}\right)}\left(\eta_{r} P_{g}+P_{b}\right) \\
& P_{t}=\left(\eta_{i} \eta_{m} \eta_{t}\right)^{\operatorname{sign}\left(\eta_{r} P_{g}+P_{b}\right)}\left(\eta_{r} P_{g}+P_{b}\right)
\end{aligned}
$$

where $\eta_{f}$ and $\eta_{m}$ depend on operating conditions, while $\eta_{r}, \eta_{d c}, \eta_{i}$ have been assumed to be constant.

Additionally, the S-HEV dynamics depends on four state variables $\mathbf{x}=\left(Q_{f}, Q_{b}, u, s\right)^{T}$, respectively the consumed fuel, battery charge, vehicle speed, and travelled distance. By manipulating equations (6), (15), (17), and (25) the following standard state space formulation is obtained:

$$
\frac{d t}{d}\left(\begin{array}{c}
Q_{f} \\
Q_{b} \\
u \\
s
\end{array}\right)=\left(\begin{array}{c}
q_{f 0}+P_{g} /\left(Q_{H V} \alpha_{f}\right) \\
P_{b} / v_{b} \\
\left(P_{t}+P_{h}\right) /(m u)-\left(F_{t}+F_{D}\right) / m \\
u
\end{array}\right)
$$

where the dependency from the braking power $P_{h}$ is now explicit. Any other variables of the system, such as electric currents and voltages, torques, etc., may be explicitly calculated as functions of state variables $\mathbf{x}$ and driving inputs u. 


\section{Green Driving Problem Formulation}

For any given value of the vehicle speed and acceleration, the necessary driving force $F_{u}$ is uniquely determined by the expression (15), however the proportion among power components $P_{g}, P_{b}$ and $P_{h}$ is undetermined and in particular it is possible to select a proper powers component combination that minimizes fuel consumption. Power management optimisation is usually conducted on the basis of minimizing fuel consumption for a given vehicle speed profile. In this work a different approach is used: the vehicle mission is specified in terms of a route which has to be completed in an assigned amount of time, in other words only the average speed is assigned, while the instantaneous speed is also optimized.

From the mathematical point of view, the optimal control problem (OCP) is to find the power inputs $\mathbf{u}$ that minimize the consumed fuel $Q_{f}(T)$ at the end of the given trip:

$$
\begin{array}{cl}
\text { minimize: } & \min _{\mathbf{u}} Q_{f}(T) \\
\text { subject to: } & \frac{d}{d t} \mathbf{x}=\mathbf{f}(\mathbf{x}, \mathbf{u}, t) \\
& \psi(\mathbf{x}, \mathbf{u}, t) \leq \mathbf{0} \\
& \mathbf{b}(\mathbf{x}(0), \mathbf{x}(T))=\mathbf{0}
\end{array}
$$

The state space model (28b) has already been specified in Equations (27). Inequality constraints (28c) are used to keep the operating conditions of the powertrain inside their admissible range, and to guarantee the driving safety and comfort.

The list of power train constraints includes the limitation of the generator power:

$$
0 \leq P_{g} \leq P_{g, \max }
$$

The battery is constrained in terms of charge and current:

$$
\begin{gathered}
Q_{b, \text { min }}<Q_{b}<Q_{b, \text { max }} \\
i_{b d}<i_{b}<i_{b c}
\end{gathered}
$$

The PMS motor (and indirectly the inverter) is constrained in terms of voltage and current:

$$
\begin{gathered}
v_{d m}^{2}+v_{q m}^{2} \leq \frac{v_{d c}^{2}}{3} \\
-i_{\text {max }} \leq i_{\text {qm }} \leq i_{\text {max }}
\end{gathered}
$$

Finally, braking power is constrained to be negative:

$$
P_{h} \leq 0
$$

Additional constraints are employed to guarantee driving safety and comfort, starting from the speed which is constrained within the legal speed limit $u_{L}$ as follows:

$$
u \leq u_{L}
$$

For driving safety, the longitudinal and lateral acceleration should be constrained in order to remain (at least) inside the ellipse of adherence of tires. However, there is some experimental evidence [28] than everyday drivers use accelerations remarkably smaller than adherence limits, moreover the acceleration envelope is not an ellipse since drivers tolerate bigger pure longitudinal/lateral accelerations than combined longitudinal/lateral accelerations. This human inclination to comfortably drive far from adherence limits may be synthesized by an acceleration diamond [28] mathematically described as follows:

$$
\left|\frac{\dot{u}}{a_{x, \text { max }}}\right|+\left|\frac{u \Omega}{a_{y, \text { max }}}\right|-1 \leq 0
$$

Boundary conditions (28d) are used to impose that the vehicle speed is null at the begin and at the end of the trip $u(0)=u(T)=0$ and that the trip will be completed in the given time $s(T)-s(0)=L$. The time $T$ can be expressed in terms of an average speed for the journey. Moreover, according to the idea that the battery should be charged without any external source of electricity, the low frequency component of the battery charge should not vary. This condition has been replaced by the imposing of the same battery charge at the beginning and end of the trip $Q_{b}(0)=Q_{b}(T)=Q_{b I}$.

The optimal control problem defined in Equations (28) may be solved using various methods [29], such as non linear programming, dynamic programming or indirect methods. In this work the indirect approach has been used and the optimization problem has been converted into a two point boundary value problem. More details on the adopted approach may be found in [19], [30].

\section{Simulation Results}

A real rural route $6 \mathrm{~km}$ long has been selected as the vehicle mission, as depicted in Figure 7. Road geometry has been taken from maps.google.it and then converted into the curvature model (19), while edges have been removed by constraining the curvature to a maximum value $|\Theta|<$ $0.12 m^{-1}$. Traffic is not considered and therefore the speed is not constrained to the behavior of other vehicles. As an example, a mission of a requested average speed of $65 \mathrm{~km} / \mathrm{h}$ has been simulated, with Figure 8 showing the results for the optimised speed and acceleration profiles. The presence of repeated manoeuvres, that correspond to an intersection (or sharp corner) followed by an (almost) straight segment and ended with another intersection, is clearly visible from this figure. With reference to four time marker points on the speed plot, one such manoeuvre starts at a low speed (at time $t_{A}$ ), which is constrained by the admissible lateral

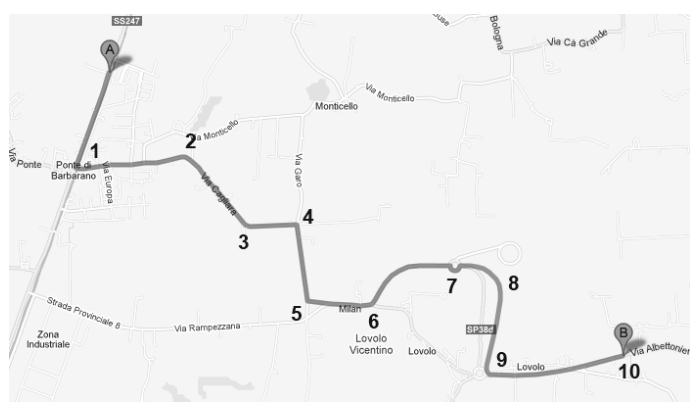

Fig. 7. Rural route selected for the vehicle mission. 

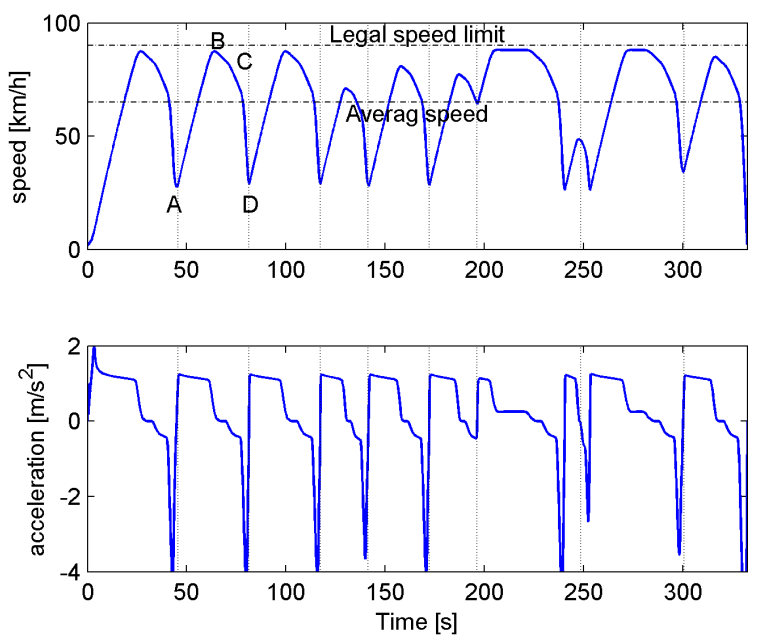

Fig. 8. Optimized vehicle speed and acceleration profiles (vertical lines correspond to the curves indicated in figure 7).
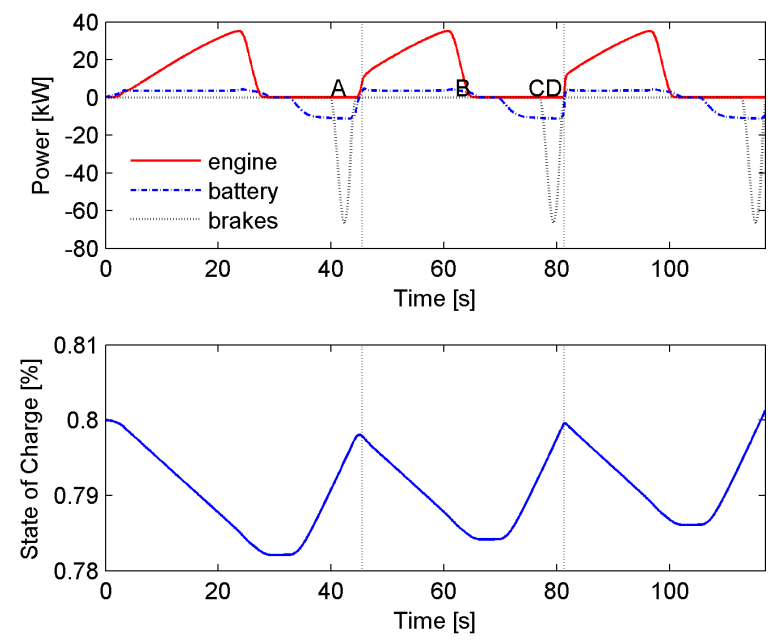

Fig. 9. Optimized power flow and battery State of Charge.

acceleration while cornering, then the vehicle accelerates to a maximum value $\left(t_{B}\right)$, but this maximum speed is not maintained for a noticeable time. On the contrary, after point $t_{B}$ the vehicle mildly decelerates to point $t_{C}$, then the deceleration is more evident up to point $t_{D}$. By looking at the power flows in Figure 9, it can be observed that in the acceleration phase $t_{A}-t_{B}$ the propulsive power is mainly provided by the SI engine, while the battery provides an additional, small amount of power. From $t_{B}$ to $t_{C}$ the used propulsive power is small and the vehicle decelerates because of friction forces (air drag, tire friction, etc). From $t_{C}$ to $t_{D}$ brakes are used to decelerate to the cornering speed $v_{D}$, in the meanwhile the battery is recharged to counterbalance the energy delivered in the phase $t_{A}-t_{B}$.

It is worth pointing out that besides the optimal power allocation, also the optimal speed profile has been calculated.

The pattern of such optimal speed profile appears to be

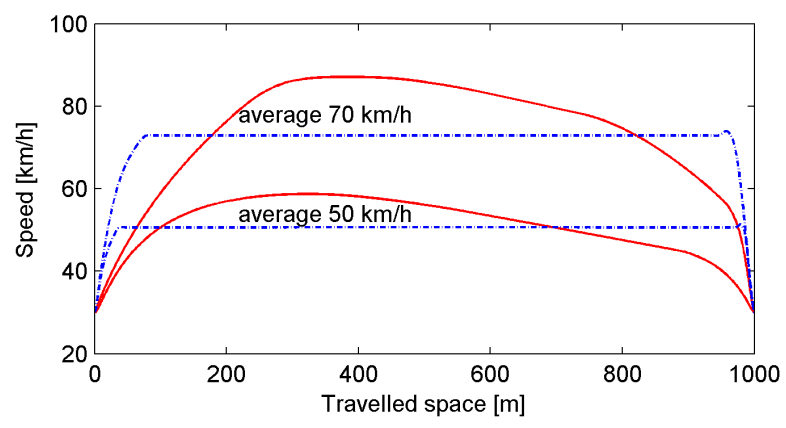

Fig. 10. Speed patterns that minimize fuel consumption (solid lines) and speed variation for regular driving style (dashed lines) for a $1 \mathrm{~km}$ straight road.

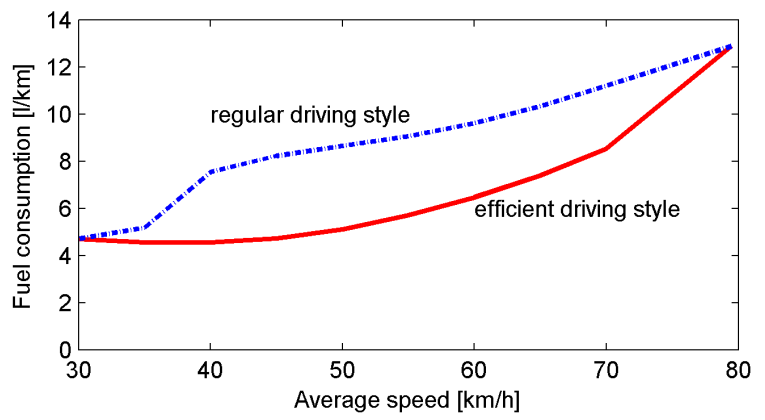

Fig. 11. Fuel consumption vs average speed for different driving styles.

different from a "normal driving" speed profile. In the latter case, the driver would reasonably adopt a more regular speed profile, i.e he/she accelerates to a cruise speed value, then keeps this value constant until the next curve approaches, when he/she decelerates. Such differences are highlighted in figure 10, which depicts speed patterns of both efficient (solid lines) and regular (dashed lines) driving styles, for a straight road $1 \mathrm{~km}$ long between two curves and for different average speeds. The efficient speed profile is nor intuitive nor easy to be reproduced by a human driver, indeed the proper identification of maximum speed points $B_{i}$ and braking points $C_{i}$ requires a long planning distance (in this example $1 \mathrm{~km}$ ).

The fuel consumption variation with average speed for the two driving styles is depicted in Figure 11, which highlights that the selection of the efficient driving style (instead of the regular one) leads to a remarkable reduction in fuel consumption and hence it is appealing. Unfortunately, a human driver would find it difficult to adopt such an efficient style in a real situation, because the driving power is continuously changing and in particular because it is difficult to identify the point $B$ from which the engine has to be set in idle condition. However, such difficulties may be overcome with the introduction of a kind of Intelligent Cruise Control that automatically estimates the optimal speed profile and assists the driver to comply with it. All the necessary information is potentially available on-board, in particular it could be integrated with a GPS navigator system, which detects in real time the vehicle position and speed, as well as the necessary information on the road geometry 
with the necessary preview of a few kilometers. Additional information on vehicle characteristics and performance, as well as driving style and preferences, may be stored offline.

\section{CONCLusions}

This paper illustrates a methodology for the optimization of a series hybrid electric vehicle based on the indirect optimal control approach. The main advantage of the presented approach is that the optimal control problem is effectively formulated by specifying the performance criterion to be optimized plus a set of constraints that must be satisfied, without the necessity of any predefined heuristic rule or control architecture. Another distinguishable feature is that the vehicle mission is not defined in terms of a given speed profile, instead for the given route the speed is optimized by complying with time, safety and comfort constraints. It has been shown that on a rural route with scarce traffic, the simultaneous optimization of the speed and power flow leads to a remarkable reduction of fuel consumption. Conversely, optimized speed patterns are counter intuitive and difficult to reproduce for a human rider. However, this difficulty would be reduced if the driver would be assisted by means of an Advanced Cruise Control that implements such an optimization strategy and assists the driver (rather than substitutes him/her). From a technological point of view this would be feasible as the presented software works in real time and requires as input only basic information on the vehicle state and route characteristics that are easily available on a navigation system. A major limitation is that intense traffic conditions, such as in urban driving, cannot be assessed with this method. Indeed, in this case the velocity of the ecovehicle is constrained by the presence of other vehicles and hence cannot be freely optimized.

\section{REFERENCES}

[1] F. R. Salmasi, "Control strategies for hybrid electric vehicles: evolution, classification, comparison, and future trends," IEEE Trans. Veh. Technol., vol. 56, no. 5, pp. 2393-2404, September 2007.

[2] D. A. Crolla, Q. Ren, S. ElDemerdash, and F. Yu, "Controller design for hybrid vehicles - state of the art review," in Vehicle Power and Propulsion Conference, 2008. VPPC '08. IEEE, sept. 2008, pp. $1-6$.

[3] X. He, M. Parten, and T. Maxwell, "Energy management strategies for a hybrid electric vehicle," in Vehicle Power and Propulsion, 2005 IEEE Conference, sept. 2005, pp. 390 - 394.

[4] A. Sciarretta, M. Back, and L. Guzzella, "Optimal control of parallel hybrid electric vehicles," Control Systems Technology, IEEE Transactions on, vol. 12, no. 3, pp. 352-363, 2004.

[5] L. V. Pérez and E. A. Pilotta, "Optimal power split in a hybrid electric vehicle using direct transcription of an optimal control problem," Math. and Comput. in Simulation, vol. 79, no. 6, pp. 1959-1970, February 2009.

[6] S. Kutter and B. Baker, "Predictive online control for hybrids: Resolving the conflict between global optimality, robustness and realtime capability," in Vehicle Power and Propulsion Conference (VPPC), 2010 IEEE, Sept. 2010, pp. 1-7.

[7] C. Musardo, G. Rizzoni, and B. Staccia, "A-ECMS: An adaptive algorithm for hybrid electric vehicle energy management," in Decision and Control, 2005 and 2005 European Control Conference. CDC-ECC '05. 44th IEEE Conference on, Dec. 2005, pp. 1816 - 1823.

[8] G. Paganelli, S. Delprat, T. M. Guerra, J. Rimaux, and J. J. Santin, "Equivalent consumption minimization strategy for parallel hybrid powertrains," in Vehicular Technology Conference, 2002. VTC Spring 2002. IEEE 55th, vol. 4, 2002, pp. 2076 - 2081 vol.4.
[9] L. Serrao, S. Onori, and G. Rizzoni, "ECMS as a realization of pontryagin's minimum principle for HEV control," in American Control Conference, 2009. ACC '09., June 2009, pp. 3964 -3969.

[10] A. B. Schwarzkopf and R. B. Leipnik, "Control of highway vehicles for minimum fuel consumption over varying terrain," Transportation Research, vol. 11, no. 4, pp. 279 - 286, 1977.

[11] V. V. Monastyrsky and I. M. Golownykh, "Rapid computation of optimal control for vehicles," Transportation Research Part B: Methodological, vol. 27, no. 3, pp. $219-227,1993$.

[12] Y. Wang, B. Ning, F. Cao, B. De Schutter, and T. J. J. Van den Boom, "A survey on optimal trajectory planning for train operations," in Service Operations, Logistics, and Informatics (SOLI), 2011 IEEE International Conference on, 2011, pp. 589-594.

[13] R. Franke, M. Meyer, and P. Terwiesch, "Optimal control of the driving of trains (optimale steuerung der fahrweise von zügen)," at - Automatisierungstechnik, vol. 50, no. 12_2002, p. 606, 2002.

[14] M. Miyatake and H. Ko, "Optimization of train speed profile for minimum energy consumption," IEEJ Transactions on Electrical and Electronic Engineering, vol. 5, no. 3, pp. 263-269, 2010.

[15] E. Hellström, M. Ivarsson, J. Åslund, and L. Nielsen, "Look-ahead control for heavy trucks to minimize trip time and fuel consumption," Control Engineering Practice, vol. 17, no. 2, pp. 245-254, 2009.

[16] T. van Keulen, B. de Jager, D. Foster, and M. Steinbuch, "Velocity trajectory optimization in hybrid electric trucks," in 2010 American Control Conference (ACC), 2010, pp. 5074-5079.

[17] A. P. Stoicescu, "On fuel-optimal velocity control of a motor vehicle," International Journal of Vehicle Design, vol. 16, no. 2/3, pp. 229-256, 1995.

[18] S. Eben Li and H. Peng, "Strategies to minimize the fuel consumption of passenger cars during car-following scenarios," Proceedings of the Institution of Mechanical Engineers, Part D: Journal of Automobile Engineering, vol. 226, no. 3, pp. 419-429, 2012.

[19] E. Bertolazzi, F. Biral, and M. Da Lio, "Symbolic-numeric efficient solution of optimal control problems for multibody systems," Journal of Computational and Applied Mathematics, vol. 185, no. 2, pp. 404$421,2006$.

[20] J. B. Heywood, "Internal combustion engine fundamentals," 1988.

[21] M. Masi and P. Gobbato, "Measure of the volumetric efficiency and evaporator device performance for a liquefied petroleum gas spark ignition engine," Energy Conversion and Management, vol. 60, pp. 18-27, 2012. [Online]. Available: www.scopus.com

[22] P. Pillay and R. Krishnan, "Modelling, simulation, and analysis of permanent-magnet motor drives, Part I: The permanent-magnet synchronous motor drive," IEEE Transactions on Industry Application, vol. 25, no. 2, pp. 265-273, 1989.

[23] J. Hey, D. A. Howey, R. Martinez-Botas, and M. Lamperth, "Transient thermal modeling of an axial flux permanent magnet (AFPM) machine using a hybrid thermal model," International Conference on Fluids and Thermal Engineering, 2010.

[24] N. Mohan, T. M. Undeland, and W. P. Robbins, Power Electronics: Converters, Applications, and Design. USA: John Wiley \& Sons, 2002.

[25] S. A. Evangelou and A. Shukla, "Advances in the modelling and control of series hybrid electric vehicles," in Proceedings of 2012 American Control Conference. Montreal, Canada: IEEE, 27-29 June 2012.

[26] C. M. Shepherd, "Design of primary and secondary cells - part 2. an equation describing battery discharge," Journal of Electrochemical Society, vol. 112, pp. 657-664, July 1965.

[27] O. Tremblay and L. A. Dessaint, "Experimental validation of a battery dynamic model for ev applications," World Electric Vehicle Journal, vol. 3, 2009.

[28] F. Biral, M. Da Lio, and E. Bertolazzi, "Combining safety margins and user preferences into a driving criterion for optimal controlbased computation of reference maneuvers for an adas of the next generation," IEEE Intelligent Vehicles Symposium, Proceedings, vol. 2005, pp. 36-41, 2005.

[29] A. E. Bryson, Dynamic optimization. Addison Wesley Longman, 1999.

[30] V. Cossalter, M. Da Lio, R. Lot, and L. Fabbri, "A general method for the evaluation of vehicle manoeuvrability with special emphasis on motorcycles," Vehicle System Dynamics, vol. 31, no. 2, pp. 113-135, 1999. 